\title{
Development of a Korean-specific cardiovascular risk model in a cohort at high-risk of hypertension
}

\author{
Il Suk Sohn
}

Department of Cardiology, Kyung Hee University Hospital at Gangdong, Seoul, Korea

Received: August 3, 2021 Accepted: August 12, 2021

\section{Correspondence to}

Il Suk Sohn, M.D.

Department of Cardiology, Kyung Hee University Hospital at Gangdong, 892 Dongnam-ro, Gangdong-gu, Seoul 05278, Korea Tel: + 82-2-440-6108

Fax: +82-2-440-7699

E-mail: issohn@khu.ac.kr https://orcid.org/0000-00018004-5185

\section{See Article on Page 1115-1125}

Cardiovascular disease (CVD) is the leading cause of death worldwide, and hypertension (HTN) increases the risk of CVD [1]. HTN, which is associated with ischemic heart disease, stroke, chronic kidney disease, and overall mortality, is one of the most important and modifiable CVD risk factors. Over the past 50 years, considerable research has been conducted to determine the optimal target blood pressure (BP), to reduce CVD risk and mortality associated with various comorbidities, including heart failure, stroke, and diabetes, and across different world regions [25]. Ethnic differences in CVD risk and mortality are well documented [6]. The risk of stroke is higher in Asian populations than in Western countries, and the relationship between BP and stroke incidence is stronger in Asian populations than in Western populations [7].

A recent landmark clinical study, the Systolic Blood Pressure Intervention Trial (SPRINT) sponsored by the United States National Institutes of Health has received considerable attention [8]. The study found that, compared with standard BP control (systolic BP < 140 $\mathrm{mmHg}$ ), treating to a lower target systolic BP of $<120 \mathrm{mmHg}$ reduced CVD incidence and mortality among patients at high risk of CVD (without dia- betes or a history of stroke), reduced the primary CVD composite outcome by $25 \%$, and reduced all-cause mortality by $27 \%$. After publication of these findings, most guidelines recommended strict $\mathrm{BP}$ control to maximally reduce the risk of CVD in high-risk patients. However, most of the patients (about 90\%) in the SPRINT study were of Caucasian or African descent and fewer than $2 \%$ were Asian. Few clinical studies have investigated the effects of intensive BP control in Asian patients. Furthermore, the SPRINT study included high-risk patients with a $15 \%$ or greater 10 -year risk of CVD based on the Framingham risk score. It is well documented that the Framingham risk score may overestimate the CVD risk in Asians [7,9]. The fact that the CVD risk differs between Western and Asian populations, and that an optimal CVD risk score based on real-world data in Asians has not been determined, point to the need for a CVD risk prediction model developed for Asian populations.

In this issue of the Korean Journal of Internal Medicine, Lee et al. [10] described the design and baseline characteristics of the Korean Hypertension Cohort (KHC) study. The study enrolled patients diagnosed with HTN from six national university hospitals between 2006 and 2011. Detailed clinical information from each hospi- 
tal was integrated with long-term follow-up data over a period of approximately 9 years obtained from the National Health Insurance System (NHIS) claims database. A non-HTN control population in a 1:10 ratio $(100,000)$ was obtained from the NHIS sample cohort using propensity score matching. The primary objective of the KHC study was to evaluate the long-term impact of intensive BP control, which maintained systolic/diastolic pressures at $<135 / 85 \mathrm{mmHg}$, and to compare the findings with the moderate control group (systolic BP, 140 to $149 \mathrm{mmHg}$ ). The study enrolled 11,083 patients with HTN (mean age, 58.8 years; males, 50.5\%) including $31.4 \%$ with never-treated HTN. In total, $32.9 \%$ of the participants were in the moderate cardiovascular risk group and $37.7 \%$ were in the high-risk group. Treatment with 1.9 tablet doses of antihypertensive medication reduced baseline BPs of $149.4 / 88.5 \mathrm{mmHg}$ to $130.8 / 78.0 \mathrm{mmHg}$ over the 2-year hospital follow-up period. Cardiovascular events occurred in $7.5 \%$ of patients overall; $8.5 \%$, $8.8 \%$, and $4.7 \%$ in the high-, moderate-, and low-risk patients, respectively. Although the information provided by this nationwide cohort study is timely and important, it has some limitations. First, the authors do not discuss the white-coat and masked HTN phenomena, which may lead to false BP readings in the clinical setting. Second, BP measurement protocols were not standardized, which the authors acknowledged. Third, a high proportion of the high-risk patients were recruited from tertiary referral university hospitals; therefore, the findings of the study may not be generalizable to all Korean patients with HTN.

As the first ever nationwide high-risk HTN cohort study, the ongoing results of the KHC study will provide important real-world data on the long-term outcomes of Korean patients with HT'N according to BP values, comorbid diseases, medication and adherence, as well as health behaviors and use of health resources. Finally, the findings of the study may help establish a CVD risk score for Korean patients with HTN that may be applicable to other Asian populations.

\section{Conflict of interest}

No potential conflict of interest relevant to this article was reported.

\section{REFERENCES}

1. Kim HC, Ihm SH, Kim GH, et al. 2018 Korean Society of Hypertension guidelines for the management of hypertension: part I-epidemiology of hypertension. Clin Hypertens 2019;25:16.

2. Williams B, Mancia G, Spiering W, et al. 2018 ESC/ESH guidelines for the management of arterial hypertension: the task force for the management of arterial hypertension of the European Society of Cardiology and the European Society of Hypertension. The task force for the management of arterial hypertension of the European Society of Cardiology and the European Society of Hypertension. J Hypertens 2018;36:1953-2041.

3. Unger T, Borghi C, Charchar F, et al. 2020 International Society of Hypertension global hypertension practice guidelines. Hypertension 2020;75:1334-1357.

4. Kim KI, Ihm SH, Kim GH, et al. 2018 Korean Society of Hypertension guidelines for the management of hypertension: part III-hypertension in special situations. Clin Hypertens 2019;25:19.

5. Lee JY, Han SH. Blood pressure control in patients with chronic kidney disease. Korean J Intern Med 2021;36:780794.

6. Meadows TA, Bhatt DL, Cannon CP, et al. Ethnic differences in cardiovascular risks and mortality in atherothrombotic disease: insights from the Reduction of Atherothrombosis for Continued Health (REACH) registry. Mayo Clin Proc 2011;86:960-967.

7. Ihm SH, Bakris G, Sakuma I, Sohn IS, Koh KK. Controversies in the 2017 ACC/AHA hypertension guidelines: who can be eligible for treatments under the new guidelines? An Asian perspective. Circ J 2019;83:504-510.

8. SPRINT Research Group, Wright JT Jr, Williamson JD, et al. A randomized trial of intensive versus standard blood-pressure control. N Engl J Med 2015;373:2103-2116.

9. DeFilippis AP, Young R, McEvoy JW, et al. Risk score overestimation: the impact of individual cardiovascular risk factors and preventive therapies on the performance of the American Heart Association-American College of Cardiology-Atherosclerotic Cardiovascular Disease risk score in a modern multi-ethnic cohort. Eur Heart J 2017;38:598-608.

10. Lee HY, Lee JY, Shin HG, et al. The Korean Hypertension Cohort study: design and baseline characteristics. Korean J Intern Med 2021;36:1115-1125. 